\title{
Double Photoexcitation of Helium in a Strong dc Electric Field
}

\author{
James R. Harries, ${ }^{1, *}$ James P. Sullivan, ${ }^{1}$ James B. Sternberg, ${ }^{1, \dagger}$ Satoshi Obara, ${ }^{1}$ Tadayuki Suzuki, ${ }^{1}$ Peter Hammond, ${ }^{2}$ \\ John Bozek, ${ }^{3}$ Nora Berrah, ${ }^{4}$ Monica Halka, ${ }^{5}$ and Yoshiro Azuma ${ }^{1}$ \\ ${ }^{1}$ Photon Factory, IMSS, KEK, 1-1 Oho, Tsukuba-shi, Ibaraki-ken, 305-0801 Japan \\ ${ }^{2}$ Department of Physics, University of Western Australia, Nedlands, Perth, WA 6907, Australia \\ ${ }^{3}$ Advanced Light Source, Berkeley, California \\ ${ }^{4}$ Department of Physics, Western Michigan University, Kalamazoo, Michigan \\ ${ }^{5}$ Embry Riddle Aeronautical University, Prescott, Arizona
}

(Received 9 August 2002; published 1 April 2003)

\begin{abstract}
We report the first experimental measurements of the effect of an applied field on the photoexcitation and autoionization of doubly excited states of helium. Ground-state photoionization spectra have been measured in the region below the $\mathrm{He}^{+}(N=2)$ threshold with static electric fields of up to $84.4 \mathrm{kV} / \mathrm{cm}$ across the interaction region. The results are compared to the theoretical calculations of Chung et al. [J. Phys. B 34, 165 (2001)], which are the only calculations available in this regime. Transitions to several states in the $N=2, n=6$ manifold are assigned, and a wealth of new structure is observed. Our data show that many more series are mixed in by the field than those predicted by theory.
\end{abstract}

In this Letter, we report the first experimental observation of the effect of a static (dc) applied electric field on the photoexcitation and autoionization of doubly excited states in helium below the $\operatorname{He}^{+}(N=2)$ threshold. This type of measurement allows the exploration of the interplay between the electron-ion interaction, electronelectron interactions, and the modification of the Coulomb well by the applied electric field. Helium represents a simple quantum three-body system dominated by electron correlation effects, and the doubly excited states have been extensively studied since they were observed in detail by Madden and Codling [1] in the first scientific experiment using synchrotron radiation.

By the early 1990's all three of the $L S$-allowed series converging on the $\mathrm{He}^{+}(N=2)$ ionization threshold at $65.4 \mathrm{eV}$ had been observed in photoionization [2]. Much more recently, it has become apparent that some of the photodoubly excited states also decay via fluorescence [3-5], and the superhigh resolution available at thirdgeneration light sources has enriched our understanding of these competing decay routes [6-8]. An important aspect of the recent measurements is that as the $N=2$ threshold is approached states of triplet character can be directly excited from the ground state $[8,9]$.

We compare our data with the Fang-Chung-Ho theoretical saddle-point complex-rotation calculations [1012], which predict intensity to be shared between a Stark manifold of states, with oscillator strength shifting away from the ${ }^{1} P^{0}$ states as a field is applied.

In contrast to the well-understood effect of an electric field on singly excited Rydberg states (see, for example, Zimmerman et al. [13]), predicting field effects on the formation of doubly excited states represents a different challenge since the applied field competes with electronelectron correlation and may also affect the competition between decay routes. This competition is strongest for states where $n \approx N$, with $n$ and $N$ the principal quantum numbers of the "outer" and "inner" electrons, respectively. Experimentally unachievable fields, however, are required to study, for example, the $n=N=2$ states of helium, and states with $N>2$ require higher resolution and have increasing numbers of contributing series. We have chosen to study states with $N=2, n>5$, where the field strengths and resolution available are sufficient to observe the electric-field effects. Photoexcitation of $\mathrm{H}^{-}$ has previously been studied in an electric field both experimentally and theoretically; see, for example, Halka et al. [14], Comtet et al. [15], and the review of the experimental work of Bryant et al. [16]. Despite being isoelectronic, the case of helium is very different from that of $\mathrm{H}^{-}$, where the spectrum is dominated more by Feshbach resonances.

We have developed an apparatus which is capable of performing photoion, vacuum ultraviolet (VUV) fluorescence photon, and metastable atom yield measurements when photoexcitation takes place in a static dc field of up to $100 \mathrm{kV} / \mathrm{cm}$.

Figure 1 shows the basic layout of our apparatus. The interaction region is formed by the overlap of the photon beam with a gas jet effusing from a single needle mounted on an $x y z$ manipulator. The needle is positioned sufficiently far away from the electrodes that there is no risk of breakdown to the needle. On either side of the interaction region are highly polished $120 \mathrm{~mm}$ diameter stainless steel plates with rounded edges, one connected to earth, the other to a high-voltage power supply. The earthed electrode has a $40 \mathrm{~mm}$ long, $5 \mathrm{~mm}$ wide slit which can be placed at any orientation. This slit allows the detection of photoions (repelled by the high-voltage plate) by a microchannel plate detector (MCP), labeled 


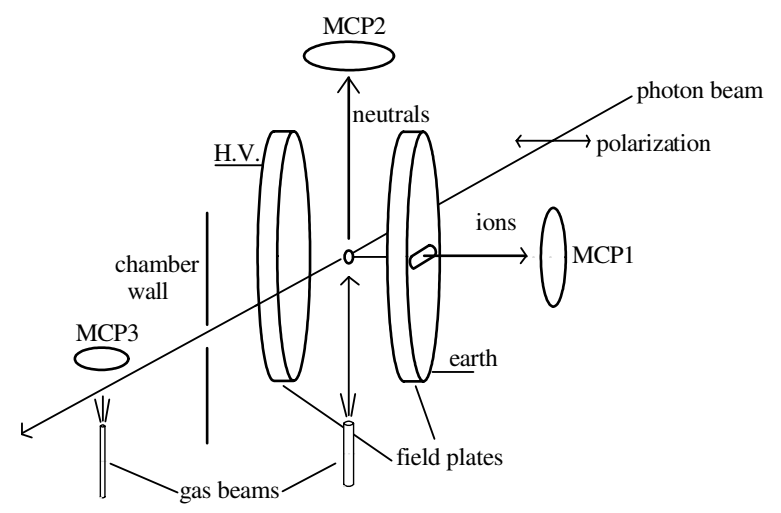

FIG. 1. Schematic diagram of the experimental setup. The electrodes have curved edges to reduce the chance of breakdowns to the chamber wall.

MCP1 in Fig. 1, and mounted just behind the earthed plate. Despite being in the direct line of sight to the highvoltage plate, the detector is well protected by the earthed electrode, and a mesh helps to prevent field penetration. A further MCP detector assembly (MCP2 in the figure) is positioned in the path of the gas jet; this detector is primarily sensitive to long-lived excited neutral atoms (metastables) and VUV photons. Charged particles can be discriminated against by using two biased meshes. By utilizing the pulsed (and gapped) timing structure of the synchrotron radiation, we are able to separate signal from the fast VUV photons and the slow metastable atoms. A third detector (MCP3 in the figure) is positioned facing a second gas jet downstream from the interaction region in a differentially pumped chamber. MCP3 can be set up to detect photoion signal or neutral signal by altering the biases on two meshes placed in front of it and provides a zero-field reference and calibration spectrum for each spectrum taken at high field in the main chamber. The polarization of the incoming radiation is parallel to the applied electric field.

The data presented here were recorded at beam line 10.0.1 at the ALS (Advanced Light Source), Berkeley, California, and preliminary measurements were made on beam lines 3B, 16B, and 20A at the Photon Factory. To resolve the Stark manifolds at the $n$ values reported here, the higher resolution and flux offered by a 3rd generation light source was essential. The data from the ALS were obtained when the synchrotron was operating in two-bunch mode and the resolving power was approximately 45000 (around $1.4 \mathrm{meV}$ at $65 \mathrm{eV}$ ). In this Letter, we present only photoionization data recorded using MCP1. High-field fluorescence and metastable spectra from MCP2 will be reported elsewhere. The electric-field plates were designed to provide a field with as small a spread as possible over the interaction region. Calculations with the CPO charged-particle optics software [17] show that with the slitted electrode the field spread across a typical interaction region (defined by the shape and profile of the photon beam) is limited to a few percent.

It is not sufficient just to measure the separation between the plates to calculate the field strength from the applied voltage, since the presence of the slit in the earthed electrode strongly affects the field distribution between the plates. We determine the field strength at the interaction region by observing the Stark effect on the singly excited helium Rydberg states. Our technique is to measure the neutral yield of metastable $1 s 2{ }^{1} S$ atoms and VUV photons in MCP2 (see Fig. 1) as the photon energy is scanned in the region of, for example, the $7^{1} P$ Rydberg state. The positions of the $n$ members of the Stark manifold are then compared with calculated energies [18-21], and an effective plate separation determined. In the experiments at ALS the effective plate separation was $10.9( \pm 0.3) \mathrm{mm}$, so, for example, applying $92 \mathrm{kV}$ across the electrodes gives a field strength of $84.4 \mathrm{kV} / \mathrm{cm}$ at the interaction region.

Recorded concurrently with each in-field photoionization spectrum was a zero-field ion or neutral particle spectrum using MCP3, which was used to provide an accurate photon energy scale calibration using state energies from Rost et al. [22]. The symmetric profiles observed in the fluorescence channel make this calibration more accurate and also make optimizing the beam line easier to give the best balance of flux and resolution. (See also [8].)

Following the notation used by Fang et al. [10], we refer to the three zero-field series converging on the $\mathrm{He}^{+}(N=2)$ threshold which are strongest in photoionization as the $(2,6 a){ }^{1} P^{0},(2,6 b){ }^{1} P^{0}$, and $(2,6 c){ }^{1} P^{0}$ series, or just $a, b$, and $c$. In the more familiar notation, the $a$ series corresponds to the "+" series, and $b$ and $c$ to the "-" and " $n d "$ series, respectively.

Figure 2 shows a portion of the photoion yield at zero field and at $84.4 \mathrm{kV} / \mathrm{cm}$, the highest field applied at the ALS. Some saturation due to high gas pressure in the differentially pumped end station (see Fig. 1) is visible in the MCP3 zero-field spectrum, which was recorded at the same time as the high-field spectrum. The doubly excited state structure is modified strongly by the field, the effect increasing with increasing $n$. The gross structure of the in-field spectra is that of a manifold of states appearing mainly at the high energy side of each zero-field $a$ state. The shift in the $6 a$ state from zero field is $5.3( \pm 0.5) \mathrm{meV}$, and the same shift measured at a field strength of $50.5 \mathrm{kV} / \mathrm{cm}$ was $1.9( \pm 0.5) \mathrm{meV}$. This compares well with values interpolated from the calculations of Chung et al. [11] of approximately 5.1 and $1.8 \mathrm{meV}$. The weak feature to the low photon energy side of the $6 a$ states in the $84.4 \mathrm{kV} / \mathrm{cm}$ spectrum of Fig. 2 is at $64.9768 \mathrm{eV}$, $23.6 \mathrm{meV}$ below the zero-field $6 a$ state. The value interpolated from the data of Fang et al. [10] for the position of the $(2,6 a){ }^{1} D^{0}$ state is $21.3 \mathrm{meV}$ below the zero-field $6 a$ state. It should be noted that our experimental data are 


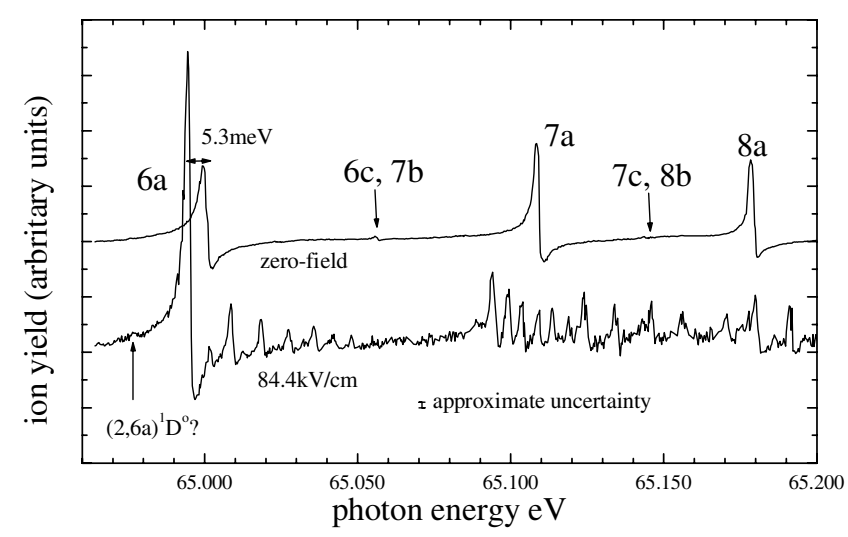

FIG. 2. Photoion spectrum at zero field and with an applied field of $84.4 \mathrm{kV} / \mathrm{cm}$ in the region of excitation to the $6 a, 7 a$, and $8 a$ states. Scales and offsets are chosen for maximum clarity. Some saturation is visible in the zero-field spectrum; see text. The statistical uncertainty of the high-field data is shown.

calibrated to the theoretical calculations of [22], whose position for the $6 a$ state is $0.7 \mathrm{meV}$ lower than that of Chung et al.

Figure 3 shows the evolution of the spectra recorded as a function of field strength between the $6 a$ and $7 a$ states. For all but the $84.4 \mathrm{kV} / \mathrm{cm}$ spectrum, the statistical uncertainty is of the order of or less than the line thickness of the plotted data. For the $84.4 \mathrm{kV} / \mathrm{cm}$ data set the statistical uncertainty is shown in Fig. 2. Also plotted are the energy positions calculated by Chung et al. for several of the $6 a, 6 b$, and $6 c$ state manifolds and two $n=7$ states. The $y$ axis corresponds to field strength, and the experimental spectra have been plotted to facilitate comparison with theory. For example, the $50.5 \mathrm{kV} / \mathrm{cm}$ spectrum is plotted such that its baseline lies near $50.5 \mathrm{kV} / \mathrm{cm}$, and the $9.17 \mathrm{kV} / \mathrm{cm}$ data set plotted so the structure near $65.060 \mathrm{eV}$ lies near $9.17 \mathrm{kV} / \mathrm{cm}$.

Many more resonances are observed in the spectra than are predicted by theory [11], appearing as narrow features on broader structures in the ion yield. These broad structures represent only partially resolved groupings of Stark states. With increasing field strength the energy positions of the states shift, the intensity distribution over the manifold changes, and the broad structures gradually flatten out. This rich structure in the spectra as compared to the theoretical calculations is well evidenced in the $9.17 \mathrm{kV} / \mathrm{cm}$ spectrum near $65.06 \mathrm{eV}$, where only four states $\left(6 c^{1} S^{e}, 6 b^{1} S^{e}, 7 b^{1} S^{e}\right.$, and $\left.7 a^{1} S^{e}\right)$ are predicted to share intensity (Fig. 3 of [12]), whereas the experimental result shows two broad structures on which is superimposed a large number of narrow features. Similar discrepancies can be seen at higher field strengths. The theory predicts two main groupings of resonances at zero field in the energy region between the $6 a$ and $7 a$ states. These states are predicted to disperse in energy as the field increases with a number of avoided crossings, for

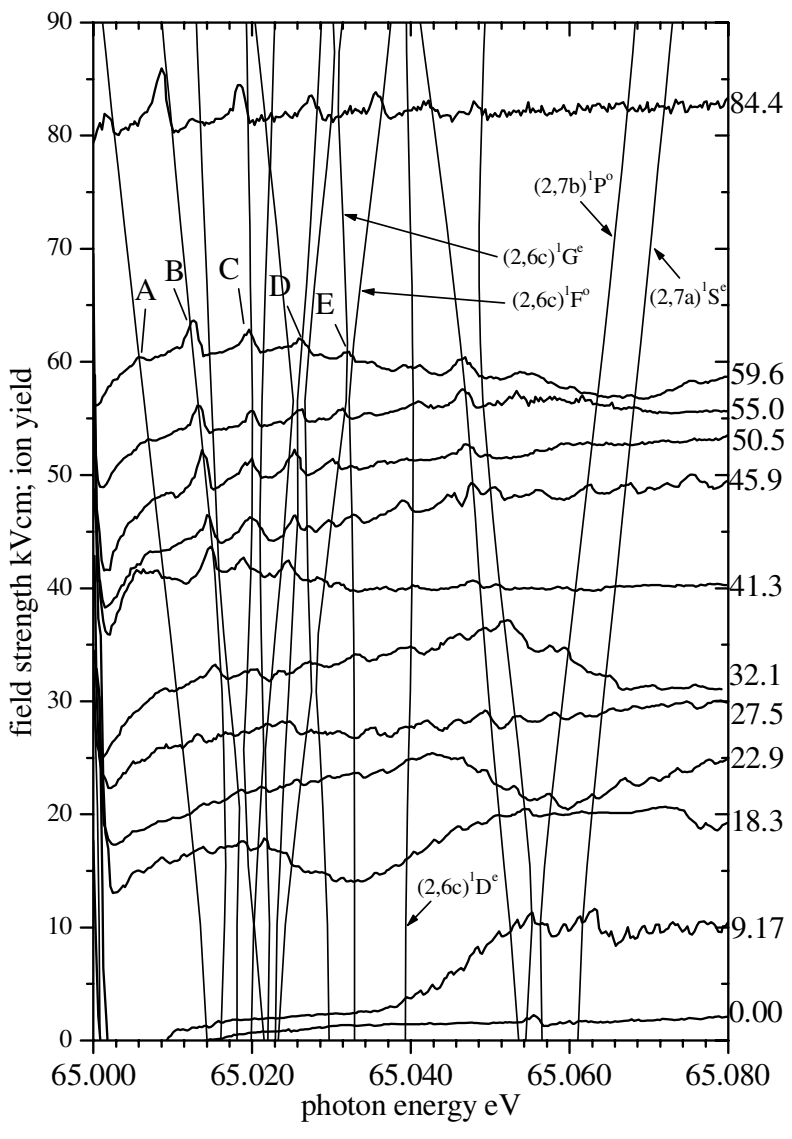

FIG. 3. Photoionization signal at various field strengths in the vicinity of the $6 a$ state. Field strengths are labeled in $\mathrm{kV} / \mathrm{cm}$ on the right-hand axis. The ion signal is normalized to the current of electrons in the storage ring and the dwell time. Theoretical state energies taken from [11] are shown and appear as vertical traces. Labels $A-E$ represent peaks whose positions are plotted as a function of field strength in Fig. 4.

example, that between the $(2,6) c^{1} G^{e}$ and the $(2,6) c^{1} F^{0}$ states near $55 \mathrm{kV} / \mathrm{cm}$. The spectra show that above about $40 \mathrm{kV} / \mathrm{cm}$ the intensity is concentrated in only a few states for each manifold, allowing individual states to be more easily identified. This intensity redistribution effect is also seen for the $n=7$ manifolds in Fig. 2 where in the high-field spectrum the resonance due to the $(2,7) a^{1} S^{e}$ state is of a similar intensity to other states of the manifold. Good agreement at higher fields is observed between some of the features in the spectrum and the theoretical predictions [12] as shown in Fig. 4. Here the more prominent features in Fig. 3 (labeled $A$ to $E$ ) have been selected and the energy positions of their peaks plotted. No allowance for asymmetric intensity profiles of features has been included because of the complexity of the spectrum. The uncertainty of the peak energy is of the order $1 \mathrm{meV}$.

From Fig. 4 we can identify some of the peaks as belonging to the Stark shifted components of the $(2,6) a$, $(2,6) b$, and $(2,6) c$ series. The peaks $A-E$ labeled in Fig. 3 can be assigned to the $(2,6) c^{1} G^{e},(2,6) b{ }^{1} G^{e},(2,6) a^{1} I^{e}$, 


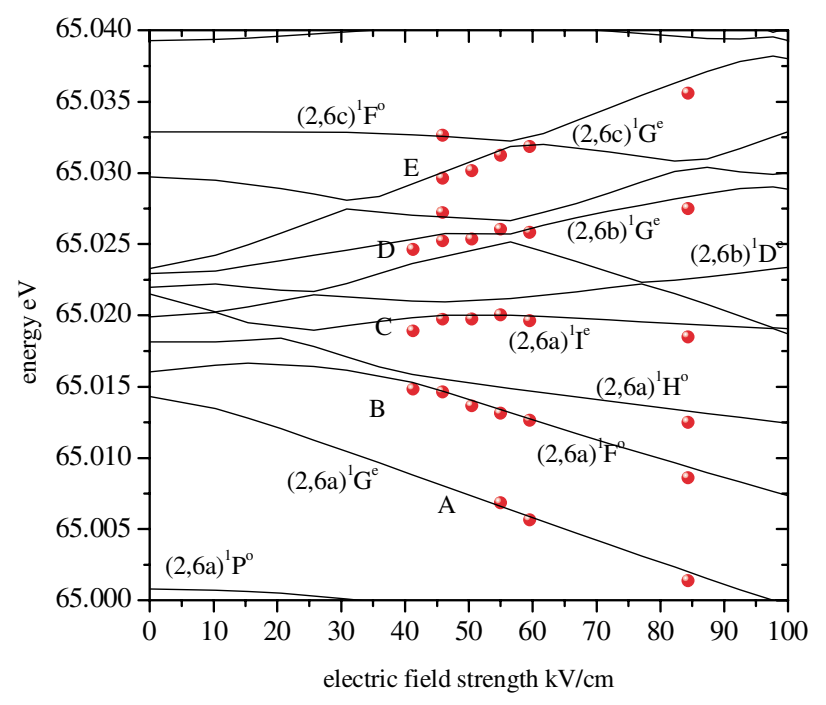

FIG. 4 (color online). Stark map showing positions of several peaks in the vicinity of the $6 a$ manifold at various field strengths. Labels $A-E$ correspond to those in Fig. 3, and theoretical energies from [11] are also shown.

$(2,6) a^{1} F^{0}$, and $(2,6) a^{1} G^{e}$ Stark states. In zero-field photoionization the resonances due to $a$ states are several times stronger in intensity than those arising from $b$ states which are in turn stronger than those from $c$ states. When a field is applied, this is no longer true.

Significant discrepancies between experiment and theory are highlighted by the present measurements, particularly for electric-field strengths below around $40 \mathrm{kV} / \mathrm{cm}$. At higher field strengths, there is more consistency between some of the observed features and theoretical predictions. It is clear from our data that the effect of an electric field on the photoexcitation and autoionization of doubly excited states in the energy region explored in these studies is not completely described by the theory of Chung et al. These discrepancies may be resolved by theoretical calculations which also include the fluorescence decay route for Stark split states and the possibility that states of triplet character may also be contributing to the spectra $[7,8]$. The unexpected complexity of the spectra in the field region below $40 \mathrm{kV} / \mathrm{cm}$ is of particular interest.

J.R.H. gratefully acknowledges the support of the Royal Society/JSPS, J.B.S. and J.P.S. that of the JSPS, and P. H. that of AMRFP/ANSTO. We thank T. K. Fang and Y. K. Ho for making their data available to us. The technical help of Tsumoru Shintake and Yuko Takasu of the KEK Accelerator Laboratory was vital during the design of the electrodes and high-voltage feedthrough.
*Email address: harries@ post.kek.jp

${ }^{\dagger}$ Current address: Department of Physics and Astronomy, University of Tennessee, Knoxville, Tennessee 37916.

[1] R. P. Madden and K. Codling, Phys. Rev. Lett. 10, 516 (1963).

[2] M. Domke, G. Remmers, and G. Kaindl, Phys. Rev. Lett. 69, 1171 (1992).

[3] E. Sokell, A. A. Wills, P. Hammond, M. A. MacDonald, and M. K. Odling-Smee, J. Phys. B 29, L863 (1996).

[4] M. K. Odling-Smee, E. Sokell, P. Hammond, and M. A. MacDonald, Phys. Rev. Lett. 84, 2598 (2000).

[5] M. Žitnik, K. Bučar, and M. Štuhec, Phys. Rev. A 65, 032520 (2002).

[6] J.-E. Rubensson, C. Såthe, S. Cramm, B. Kessler, S. Stranges, R. Richter, M. Alagia, and M. Coreno, Phys. Rev. Lett. 83, 947 (1999).

[7] T.W. Gorczyca, J. E. Rubensson, C. Såthe, M. Ström, M. Agåker, D. Ding, S. Stranges, R. Richter, and M. Alagia, Phys. Rev. Lett. 85, 1202 (2000).

[8] F. Penent, P. Lablanquie, R. Hall, M. Žitnik, K. B̌ucar, S. Stranges, R. Richter, M. Alagia, P. Hammond, and J. Lambourne, Phys. Rev. Lett. 86, 2758 (2001).

[9] A. A. Wills, E. Sokell, T.W. Gorczyca, X. Feng, M. Wiedenhoeft, S.E. Canton, and N. Berrah, J. Phys. B 35, L373 (2002).

[10] T. K. Fang and Y. K. Ho, Phys. Rev. A 60, 2145 (1999).

[11] K. T. Chung, T. K. Fang, and Y. K. Ho, J. Phys. B 34, 165 (2001).

[12] T. K. Fang and K. T. Chung, J. Phys. B 34, 1245 (2001).

[13] M. L. Zimmerman, M. G. Littman, M. M. Kash, and D. Kleppner, Phys. Rev. A 20, 2251 (1979).

[14] M. Halka, P. G. Harris, A. H. Mohagheghi, R. A. Reeder, C. Y. Tang, H. C. Bryant, J. B. Donahue, and C. R. Quick, Phys. Rev. A 48, 419 (1993).

[15] G. Comtet, C. J. Harvey, J. E. Stewart, H. C. Brant, K. B. Butterfield, D. A. Clark, J. B. Donahue, P. A. M. Gram, D.W. MacArthur, V. Yuan, W.W. Smith, and S. Cohen, Phys. Rev. A 35, 1547 (1987).

[16] H. C. Bryant, J. Chin. Chem. Soc. (Taipei) 48, 333 (2001).

[17] F. H. Read and N. Bowring, Charged-Particle Optics Software, http://www.electronoptics.com.

[18] J.W. Cooper and E. B. Saloman, Phys. Rev. A 26, 1452 (1982).

[19] B. N. Ganguly, J. R. Shoemaker, B. L. Preppernau, and A. Garscadden, J. Appl. Phys. 61, 2778 (1987).

[20] J. S. Foster, Proc. R. Soc. London Sect. A 117, 137 (1928).

[21] J. E. Lawler and D. A. Doughty, Adv. At. Mol. Opt. Phys. 34, 171 (1994).

[22] J. M. Rost, K. Schulz, M. Domke, and G. Kaindl, J. Phys. B 30, 4663 (1997). 